\title{
Jorge Luis Rodríguez, Teoría analítica del Derecho
}

(2021) Marcial Pons

Madrid, $821 \mathrm{pp}$.

\author{
Miguel Fernández Núñez \\ Universidad de Génova y Universidad de Girona \\ ORCID ID: 0000-0001-7870-1817 \\ miguel.fernandez.nunez@,edu.unige.it
}

Cita recomendada:

Fernández Núñez, M. (2021). Jorge Luis Rodríguez, Teoría analítica del Derecho. Eunomía. Revista en Cultura de la legalidad, 21, pp. 465-471

doi: https://doi.org/10.20318/eunomia.2021.6376

Recibido / received: 03/09/2021

La publicación de una nueva obra de Jorge Luis Rodríguez, escrita en colaboración o en solitario, constituye un evento que todo investigador de filosofía del derecho debe saludar con entusiasmo ${ }^{1}$. En esta ocasión, se trata de un evento excepcional pues, como procuraré mostrar en estas páginas, la que ahora aparece es una obra mayor para la disciplina.

El título de la monografía es toda una declaración de intenciones. Una declaración, en cualquier caso, descriptivamente adecuada. Efectivamente, Teoría analítica del derecho consiste en el examen de un conjunto amplio y sumamente articulado de problemas fundamentales para la teoría general del derecho, encarados con un enfoque propio de la filosofía analítica. Varias tesis centrales de índole teórica

\footnotetext{
${ }^{1}$ Amén de más de cincuenta artículos y capítulos de libro publicados, pueden contarse las siguientes monografías del profesor de la Universidad de Mar del Plata: Lógica de los sistemas jurídicos (2002); Relevancia normativa en la justificación de las decisiones judiciales, junto con Juan Carlos Bayón (2003); Estudios sobre interpretación y dinámica de los sistemas constitucionales, en colaboración con Claudina Orunesu y Pablo Perot (2005); Jerarquías normativas y dinámica jurídica, en colaboración con Jordi Ferrer Beltrán (2011); Deontic Logic and Legal Systems, en colaboración con Pablo Navarro (2014); Teoria del diritto e analisi logica (2014); Estructura y coherencia de los sistemas jurídicos, en colaboración con Giovanni Battista Ratti, de próxima aparición.
} 
y metodológica vertebran el libro. En primer lugar, el profesor argentino aboga por el positivismo jurídico como la más apta caracterización del derecho -y, en concreto, por el positivismo excluyente como la comprensión más adecuada de los criterios de identificación del derecho- y presenta una defensa del escepticismo moral como mejor teoría metaética -identificando el escepticismo con el no cognoscitivismo, es decir, con la tesis según la cual los juicios morales expresan actitudes proposicionales distintas de las creencias y, por tanto, carecen de valor de verdad. Las dos tesis, estrechamente vinculadas entre sí, avalarían la pretensión teórica del libro de delimitar el derecho respecto de otros dominios normativos. En segundo lugar, el trabajo se enfoca muy particularmente en las cuestiones del lenguaje del derecho (un peculiar lenguaje técnico) y del razonamiento jurídico; en efecto, el autor sugiere que «muchos de los problemas que siempre han ocupado a los estudiosos del derecho giran en torno al modo en que el lenguaje del derecho crea razones para actuar» ( $p$. 17). Finalmente, también es destacable en el conjunto del libro el empleo de la lógica como instrumento con el que desentrañar problemas jurídicos.

Me detendré después en algunos propósitos prácticos y méritos del libro, pero antes conviene reconstruir con cierto grado de detalle su estructura y su contenido.

La obra se compone de dos secciones («De las normas a los sistemas jurídicos» y «Dinámica jurídica, interpretación y aplicación del derecho»), con un total de siete capítulos, cada uno de los cuales consta de cinco puntos. Como he sugerido antes, los capítulos conforman un conjunto significativamente acabado; no obstante, cada uno de ellos tiene relativa autonomía temática y de contenido. En cualquier caso, el aparato conceptual que despliegan es presentado, en gran medida, de forma progresiva y cumulativa, lo cual ofrece un aprendizaje gradual de los instrumentos propios del iusfilósofo analítico, y las remisiones de contenido entre capítulos son frecuentes, lo que permite, entre otras cosas, dar profundidad a las argumentaciones y evaluar su envergadura. Es importante señalar, finalmente, que Rodríguez diferencia en los cinco puntos que integran los capítulos un contenido más básico (en los tres primeros puntos) y uno más especializado (en los dos últimos).

Un panorama de la estructura argumental de la obra o, más bien, la cata de algunos de los principales puntos nodales puede dar cuenta, pequeña cuenta, del contenido del libro y de su importancia.

El primer capítulo está dedicado al concepto de norma y a algunas cuestiones fundamentales relativas a las normas en los planos lingüístico y lógico. Abre el libro a mi entender, muy acertadamente- una clasificación de las funciones del lenguaje y una exposición de la teoría de los actos de habla. Seguidamente, el autor examina algunas taxonomías de normas, y, en concreto, muestra su preferencia por la que, a partir de Von Wright, discrimina entre prescripciones (normas dirigidas a influir en la conducta del sujeto normativo), reglas determinativas (reglas que definen una actividad humana) y normas técnicas (reglas que establecen los medios que deben usarse para obtener un cierto fin). También se introduce la distinción de formulaciones de normas (enunciados lingüísticos), normas (el significado de tales enunciados) y proposiciones normativas (proposiciones referidas a dichos enunciados), una distinción básica pero determinante para la resolución de varios problemas afrontados a lo largo de toda la obra. En los últimos tres puntos se estudia la relación entre las normas y la lógica. Se encara el dilema de Jørgensen, relativo a la posibilidad de una lógica de normas y, tras exponer los dos cuernos de la cuestión, Rodríguez se decanta por el segundo y, en concreto, por una concepción no cognoscitiva de las normas como la única postura compatible con el escepticismo en metaética. Finalmente, se abordan algunas cuestiones que surgen de distinguir una lógica de normas y una 
lógica de proposiciones normativas; una lógica, esta última, que no está expuesta al conocido dilema.

El segundo capítulo está centrado en la caracterización del derecho y, más concretamente, en la relación entre el derecho y la moral a la luz del debate entre iusnaturalismo y positivismo jurídico. La plurisecular controversia es entendida como fruto de la tensión entre dos rasgos del derecho: su carácter social (i.e., la existencia del derecho depende, en parte, de ciertos hechos humanos y la finalidad del derecho es regular la conducta de un grupo social) y su carácter normativo (i.e., las normas jurídicas ofrecen razones para la acción). La propensión por uno u otro de estos rasgos determinaría la afiliación al positivismo jurídico (de enfatizarse el carácter social del derecho) o al iusnaturalismo (de remarcarse el carácter normativo del derecho). Asimismo, se listan distintas perspectivas en que pueden emerger diferentes versiones de iusnaturalismo y de iuspositivismo y, por ende, resultados distintos en el enfrentamiento dialéctico entre ambas familias de teorías: en el plano metaético, en el concepto del derecho, y en el plano normativo. Es en la segunda variable, en el plano conceptual, donde hay que situar la genuina contraposición y aquí el positivismo contaría con argumentos más sólidos en su defensa. Dicho esto, aunque la disputa no se produzca en el plano metaético y aunque el positivismo metodológico no tenga por qué estar comprometido con ninguna posición metaética -contrariamente al iusnaturalismo, necesariamente comprometido con alguna forma de cognoscitivismo-, ello no obsta para que se plantee un listado, solvente y comprensivo, de posturas metaéticas. Pero antes que eso se exponen las principales tesis del iusnaturalismo (la tesis de la conexión necesaria entre derecho y moral, propia del iusnaturalismo clásico, y algunas tesis del iusnaturalismo contemporáneo) y del iuspositivismo (las tesis de las fuentes sociales, de la separación conceptual entre derecho y moral, de los límites del derecho), tras lo cual se confrontan el positivismo excluyente y el positivismo incluyente. También es presentada junto con las principales tesis del positivismo una tesis apócrifa, que Rodríguez llama "tesis del legalismo" -según la cual las normas del derecho deben ser obedecidas en todas las circunstancias-, indebidamente atribuida al positivismo por algunos de sus críticos. Una conclusión general es que es posible describir el derecho desde un discurso de segundo nivel valorativamente neutral; es posible, como sostiene la denominada por Rodríguez «tesis de la neutralidad», identificar el derecho sin efectuar una evaluación moral de su contenido.

El capítulo tercero está enfocado en las normas jurídicas (más concretamente, en los proyectos reduccionistas de identificación de normas jurídicas) y en los conceptos jurídicos básicos. Tras un esbozo de las teorías imperativistas clásicas, se reconstruye la teoría kelseniana de las normas y se evalúan algunos problemas cruciales de esta vinculados con la noción de sanción, a la cual la teoría kelseniana pretende reducir toda noción jurídica fundamental. La correcta delimitación de prescripciones y reglas determinativas y la caracterización de las normas que confieren poderes como reglas del segundo tipo reviste importancia en el capítulo. Junto con la de Kelsen, se examinan otras reconstrucciones de conceptos jurídicos básicos, en ámbito anglófono (de Bentham y Hohfeld, así como sus ajustes por parte de Ross y Lindahl, entre otros), para alcanzar una reconstrucción alternativa, que parte de algunas observaciones hartianas y conjuga una base hohfeldiana y kelseniana. Esta propuesta tiene, entre otros, el rasgo distintivo relevante de emplear la obligación como noción de referencia.

El cuarto capítulo estudia el derecho en cuanto sistema normativo. Se afrontan, adoptando la teoría kelseniana como principal marco de referencia, las relaciones entre normas (las relaciones de derivación dinámica y estática), al igual que tres propiedades necesarias de todo sistema jurídico: la estructura jerárquica, la 
unidad lógica y la completitud. Se examina la noción de "validez», la ambigüedad del término, y se señala la confusión entre varios de sus significados como fuente para la doctrina kelseniana de la norma fundante básica. Rodríguez extrae la conclusión de que la norma fundante básica no puede servir como respuesta a la pregunta acerca de la cadena de validez de las normas del sistema, pues, desde un punto de vista jurídico, tal norma carece de fuerza obligatoria (no hay otra norma que imponga el deber de obedecerla), y, desde un punto de vista moral, se trataría de una pregunta sobre el deber moral de obedecer al derecho. A la solución kelseniana le quedaría una dosis de razón y es que nada de lo dicho obsta para referirse a la validez de la primera constitución histórica en términos de pertenencia al sistema, aunque en ese caso la norma que identifica a la constitución como parte del sistema no habría de ser entendida como regla de conducta -como pretendería Kelsen- sino como regla determinativa.

Si el capítulo cuarto aborda algunos elementos de la nomoestática, la nomodinámica es afrontada en detalle en el capítulo quinto. Allí, la relación entre sistemas estáticos y sistemas dinámicos (entendidos como aproximadamente equivalentes a lo que Raz denomina «sistemas momentáneos» y «no momentáneos», respectivamente) es examinada a través de dos modelos: el de la inclusión (en cuya virtud, los sistemas momentáneos son subconjuntos del sistema no momentáneo) y el de la pertenencia (para el que los sistemas momentáneos son elementos que pertenecen al sistema no momentáneo). Se privilegia esta última comprensión, de tal suerte que se concibe el sistema no momentáneo como una secuencia de sistemas momentáneos que se suceden en el tiempo. Con esta base, se trata de señalar los criterios de identidad de un orden jurídico y se distingue entre un orden jurídico depurado y un orden jurídico no depurado, resultantes de dos comprensiones de la operatoria de la lex superior como criterio de preferencia entre normas: si se entiende que el criterio de lex superior actúa ex ante, bloqueando el ingreso de normas incompatibles con otras de una fuente de derecho jerárquicamente superior, entonces no se producen inconsistencias entre normas jerárquicamente diferentes en ninguno de los sistemas que conforman el orden depurado; si, en cambio, se considera que el criterio de lex superior actúa ex post entonces todo acto de promulgación normativa formalmente válido podrá dar entrada a un nuevo sistema potencialmente inconsistente con la secuencia dinámica del orden no depurado. A la luz de semejantes ajustes, se examinan distintos rasgos del funcionamiento dinámico de sistemas normativos estructurados jerárquicamente.

El capítulo sexto estudia la interpretación y algunos problemas conexos. Se critican diferentes concepciones de la interpretación y se dirigen objeciones tanto a la tesis, de matriz dworkiniana, de la única respuesta correcta como a la tesis, defendida por ciertos realismos jurídicos, de la indeterminación radical del derecho. Frente a tales posiciones se defiende la tesis de la indeterminación parcial del derecho: pese a que la mayoría de las formulaciones lingüísticas sea ambigua y las normas resultantes sean imprecisas, podría haber algunos casos claros de aplicación de las normas jurídicas, en que el significado de las formulaciones normativas puede identificarse a partir de convenciones semánticas prevalecientes en cierta comunidad jurídica. Por tanto, Rodríguez propone lo que con léxico de Guastini podría llamarse una teoría ecléctica de la interpretación (ecléctica de las teorías cognoscitivas y escépticas), en virtud de la cual hay casos fáciles, en los que la interpretación es una operación puramente cognoscitiva y para los cuales el juzgador dispone de una respuesta correcta, y casos problemáticos, en los que la interpretación es una operación decisoria, y para los cuales los tribunales gozan de discrecionalidad.

El capítulo séptimo encara la cuestión del razonamiento jurídico y la justificación de las decisiones judiciales. El capítulo aborda algunos temas 
fundamentales para la disciplina, como el problema de la normatividad del derecho, pero también resulta sumamente interesante como reconstrucción y examen crítico de algunas cuestiones clásicas a través de claves de lectura contemporáneas. Así, se retoma la clásica distinción de Wróblewski entre justificación interna y externa de una decisión, para afirmar que de ella caben dos lecturas: según la primera interpretación, está internamente justificada la decisión que se sigue de las premisas seleccionadas y está externamente justificada la decisión si sus premisas son correctas; según la segunda interpretación, una decisión está internamente justificada si se deriva del derecho vigente y está externamente justificada si su contenido es moralmente admisible. En la primera interpretación, la distinción está estrechamente vinculada con la distinción entre casos fáciles y casos difíciles y el diferente modo de justificar una decisión en uno u otro caso. Si semejante lectura es acertada entonces es incorrecto, como pretenderían algunos partidarios de la teoría de la argumentación jurídica, sostener que en los casos fáciles basta con que la decisión esté internamente justificada, mientras que en los difíciles esta tiene que estar también externamente justificada. Esta solución descansaría en una ambigüedad de «justificación», término empleado para referirse bien a la validez bien a la solidez de un razonamiento (es decir, sería sólido aquel razonamiento que, además de ser válido, se sigue de premisas verdaderas o correctas). Pues bien, resulta que si por «razonamiento justificado» entendemos "razonamiento válido» una decisión estará justificada tanto en casos fáciles como en casos difíciles e, igualmente, si por «razonamiento justificado» entendemos «razonamiento sólido» la justificación debería exigirse tanto de los casos difíciles como de los fáciles.

Aunque este breve recorrido puede dar una idea del contenido de Teoría analítica del derecho y de su significación, es oportuno pronunciarse explícitamente sobre algunos propósitos y los méritos de la obra.

Estamos ante un formidable tratado y un excelente manual. Tal vez podría afirmarse, de forma algo genérica, que, para el ámbito académico contemporáneo, muchas de las propiedades que solemos estimar buenas en un manual coinciden con aquellas que tendemos a apreciar en un tratado. También es cierto, no obstante, que a un manual solemos reclamarle algunos requisitos singulares, a razón de una cierta especialidad tanto en lo relativo al público al que va dirigido (fundamentalmente, estudiantes) como a los fines que con él pretenden lograrse (eminentemente didácticos). Examinaré primero distintas cualidades que son predicables del libro bajo cualquiera de los dos géneros editoriales referidos, para evaluar luego algunos méritos y unos pocos inconvenientes -a mi entender, perfectamente salvables- del libro en calidad de manual -un potencial empleo que, además, prevé el propio autor.

Teoría analítica del derecho combina esfuerzos reconstructivos y propuestas de gran originalidad. Por una parte, se trata de una suma del saber iusfilosófico de los últimos tres siglos, que concita admirablemente profundidad y síntesis. En el libro se encuentran expuestos y analizados críticamente muchos de los principales temas clásicos de teoría del derecho. Son tratados, por citar algunos ejemplos notables, la plurisecular controversia entre iusnaturalismo y positivismo jurídico, la teoría kelseniana de las normas, o el problema de la normatividad del derecho. Por otra parte, se trata de un libro original tanto en muchos de los enfoques que adopta como en muchas de las soluciones que propone. En lo que hace a los enfoques, es muy destacable la manera en que diferentes temas tradicionales son reexaminados a la luz de nuevas propuestas: piénsese en la aplicación del instrumental analítico de sistemas normativos propio de Alchourrón y Bulygin, con frecuencia refinado por Rodríguez, o en la aplicación de una literatura actualizada de filosofía del lenguaje para la resolución de múltiples problemas de teoría del derecho, algunos de ellos indudablemente clásicos. El capítulo séptimo, dedicado al razonamiento jurídico y a 
la justificación de las decisiones judiciales, ejemplifica muy claramente este punto. En lo que se refiere a las soluciones, en Teoría analítica del derecho se vuelcan y desarrollan diferentes contribuciones elaboradas por el autor en las últimas dos décadas en campos como la lógica deóntica, la comprensión del derecho como sistema normativo y la teoría de los conceptos fundamentales. Aunque la interpolación de cuestiones y tratamientos clásicos con cuestiones y tratamientos originales o sumamente recientes constituye una constante en la obra, es cierto que hay algo así como un reparto de contenido entre los distintos puntos que conforman los capítulos. Así, entrando ya en la valoración del libro como manual, los tres primeros puntos de cada capítulo muestran una mayor propensión a ocuparse de cuestiones clásicas, más acostumbradas en los debates de juristas y teóricos del derecho y, por tanto, más imprescindibles para el aprendiz de iusfilósofo analítico, mientras que los últimos dos puntos tienden a tratar cuestiones más originales 0 recientes.

En este sentido, conviene recordar que los capítulos muestran una cierta diferenciación en cuanto al contenido tratado en los tres primeros y aquel abordado en los dos últimos puntos y que tal discriminación puede considerarse relevante de cara a la adopción del libro como manual. En palabras de Rodríguez, «los tres primeros están destinados a ofrecer una presentación del problema que se estudia en cada caso sin presuponer conocimientos previos, en tanto que los dos últimos se orientan a quienes deseen profundizar en ellos, de modo que la obra puede utilizarse tanto para cursos de grado como para estudiantes de posgrado y profesionales del derecho en general» (contraportada).

Asimismo, me parece que la diferencia entre los puntos o, mejor, la mayor especialización de los dos últimos puntos no se agota en que estos presentan un contenido más especializado, o, como he sugerido dos párrafos antes, más original o reciente, sino que hay también un cierto cambio en la formulación lingüística, en el tono, y en el modo en que el contenido es tratado. De este modo, puede apreciarse que en los tres primeros puntos el autor se muestra especialmente atento a mantener una linealidad en la presentación de la argumentación y de los elementos fundamentales del problema abordado, mientras que en los dos últimos puntos cobran singular importancia las matizaciones, las objeciones y, en general, el pormenorizado examen de los caminos por los que procede el argumento.

Creo, en suma, aunque esta no pase de ser una generalización, que los primeros tres puntos tienen un carácter más fundamental (más introductorio, si se quiere), más indispensable desde el punto de vista temático (por encarar cuestiones más clásicas y básicas) y reciben un tono y un tratamiento más lineal que los últimos dos puntos, lo cual hace recomendable restringirse exclusivamente a ellos si quiere emplearse el libro como manual para estudiantes de grado. A estas razones cualitativas para efectuar la restricción se puede añadir una de carácter cuantitativo, y es que en cada capítulo los primeros tres puntos ocupan cerca de medio centenar de páginas, lo que arroja un total de cuatrocientas páginas como potencial manual de grado; una cantidad bastante manejable con respecto al total de ochocientas veintiuna páginas que conforma el libro.

Dicho esto, creo que todavía puede haber quien piense que algunos elementos adicionales hacen difícil adoptar, sin otras precauciones, el libro reseñado -o, mejor, las cuatrocientas páginas a las que acabo de referirme- como manual. Aunque el aparato conceptual es introducido y desarrollado con gran pulcritud expositiva, resulta muy sofisticado y el léxico correspondiente, muy refinado. Los temas son tratados, a veces, con cierta complejidad y con un elevado grado de abstracción. Las dificultades de forma y de contenido, naturalmente, convergen: el 
tejido argumental, magistralmente trabado por Rodríguez, está formulado de manera sumamente precisa, de modo que la imprecisión en su reconstrucción resultaría especialmente fatal para el éxito del argumento. No me parece, en todo caso, que las que acabo de señalar sean dificultades mayores y no veo cómo puede prescindirse de ellas sin sacrificar la profundidad y riqueza del trabajo.

El juicio de que se trata de un libro algo exigente con su lector e inusualmente incisivo puede extraerse de otros elementos. Un rasgo llamativo de la estructura de Teoría analítica del derecho es que cada capítulo se abre con un caso práctico de Derecho positivo, y no se plantea su resolución inmediatamente, sino que hay que esperar unas cuantas decenas de páginas para que, al hilo de los instrumentos teóricos desplegados en el capítulo, se brinden varias claves con las que comprender los problemas teóricos que se encuentran detrás. La presentación diferida de la resolución y el hecho de que esta descanse en un aparato filosófico, a veces, muy abstracto, puede sorprender al estudiante de derecho -aunque la sorpresa puede, en muchas ocasiones, ser un gran catalizador retórico-, pero plasma una forma muy sofisticada de entender la ayuda del filósofo a las tareas del jurista. Y, lo que quizás sea igual de relevante, responde bien al espíritu del libro de desentrañar presupuestos y analizar las consecuencias de asumir ciertas comprensiones y precomprensiones.

En esta óptica, me gustaría terminar señalando un elemento distintivo muy destacable de la obra, que consiste en su esfuerzo en exponer y evaluar réplicas y contrarréplicas, en aras de obtener el mejor argumento posible. Este resultado se obtiene, entre otras cosas, con el examen concienzudo y honesto de argumentaciones poco acordes con las sostenidas por Rodríguez o, todavía, de nombres no especialmente apreciados por los autores que defienden las posturas asumidas por Rodríguez. Ello resulta manifiesto si se constata la gran cantidad de argumentos de Dworkin que son reconstruidos y evaluados en el libro, sobre muy distintas cuestiones: contra el positivismo jurídico, contra lo que el profesor oxoniense denomina el "escepticismo moral externo", contra el escepticismo interpretativo, en torno a la distinción entre principios y reglas, en torno a la distinción entre casos fáciles y difíciles.

Teoría analítica del derecho es, en suma, un examen de buena parte de las mejores argumentaciones de que disponen los teóricos del derecho, aunado a una incesante voluntad de perfeccionarlas y de encontrar otras todavía mejores. 